Check for updates

Cite this: RSC Adv., 2017, 7, 35394

Received 5th May 2017

Accepted 10th July 2017

DOI: 10.1039/c7ra05061e

rsc.li/rsc-advances

\section{Synthesis and characterization of a new ion- imprinted polymer for the selective separation of thorium(Iv) ions at high acidity}

\begin{abstract}
Hele Liang, ${ }^{a}$ Qingde Chen, (D) *a Jingyuan Ma, ${ }^{\mathrm{b}}$ Yuying Huang ${ }^{\mathrm{b}}$ and Xinghai Shen*a
A new ion-imprinted polymer (IIP) was synthesized by thermal copolymerization of bis(2methacryloxyethyl) phosphate as functional ligand and ethylene glycol dimethacrylate as cross-linker in the presence of $\mathrm{Th}^{4+}$ as a template ion. The molar ratio of the functional ligand to $\mathrm{Th}^{4+}$ was optimized to be 4 by ${ }^{31} \mathrm{P}$ NMR titration, elemental analysis, and EXAFS, which was verified by comparing the adsorption capacities and selectivities of IIPs with different composition. Thorium(IV) ions were leached out using $0.1 \mathrm{~mol} \mathrm{~L}^{-1}$ ethylenediaminetetraacetic acid disodium salt and the prepared IIP was characterized by infra-red spectroscopy, thermogravimetric analysis and Brunauer-Emmett-Teller surface area measurement. It was found that the IIP could extract $\mathrm{Th}^{4+}$ from high acidity solution. The maximum adsorption capacity was as high as $33.3 \mathrm{mg} \mathrm{g}^{-1}$ in $1.0 \mathrm{~mol} \mathrm{~L}{ }^{-1} \mathrm{HCl}$. Even in $6.0 \mathrm{~mol} \mathrm{~L}^{-1} \mathrm{HCl}$, its adsorption capacity was still considerable. Moreover, the IIP exhibited good selectivity towards $\mathrm{Th}^{4+}$, especially in the presence of competing ions such as $\mathrm{La}^{3+}, \mathrm{Eu}^{3+}, \mathrm{Yb}^{3+}, \mathrm{UO}_{2}^{2+}$, and $\mathrm{Fe}^{3+}$.
\end{abstract}

\section{Introduction}

Thorium is widely used in various areas, such as optics, radio, aeronautics, and the chemical industry. ${ }^{1}$ More importantly, thorium is a promising fuel for the next generation of nuclear power plants. ${ }^{2}$ In general, thorium, uranium, rare-earth elements and iron often coexist in minerals as well as in waste water. ${ }^{3,4}$ Because thorium has both chemical toxicity and radioactivity, ${ }^{5}$ it is necessary to detect and remove $\mathrm{Th}^{4+}$ in waste water. Up to now, many methods have been applied, such as liquid-liquid extraction, ${ }^{6}$ solid phase extraction (SPE), ${ }^{7}$ ion exchange, ${ }^{8}$ extraction chromatography, ${ }^{9}$ membrane separation $^{10}$ and so on. Among all the methods, SPE is widely used because of flexible working conditions and simple procedures. In the field of SPE, it is most important to synthesize an adsorbent with high selectivity towards the target ion. ${ }^{11}$

Ion-imprinted polymers (IIPs) are a new kind of adsorbent for SPE, and are usually prepared by the copolymerization of a functional ligand and a cross-linker in the presence of template ion. ${ }^{12-14}$ Because of its memory effect on the template ion, the IIP can extract the target ion with good selectivity. Therefore, IIP is often used to separate and pre-concentrate

\footnotetext{
${ }^{a}$ Beijing National Laboratory for Molecular Sciences (BNLMS), Fundamental Science on Radiochemistry and Radiation Chemistry Laboratory, Department of Applied Chemistry, College of Chemistry and Molecular Engineering, Peking University, Beijing 100871, P. R. China. E-mail: qdchen@pku.edu.cn; xshen@pku.edu.cn; Tel: +86-10-62755200; +86-10-62765915

${ }^{b}$ Shanghai Synchrotron Radiation Facility, Shanghai Institute of Applied Physics, Chinese Academy of Sciences, Shanghai 201800, P. R. China
}

trace metal ions. ${ }^{15,16}$ In the preparation of IIP, understanding the formation of complex compounds between metal ions and functional ligands is very helpful. Thus, many researchers studied the interaction between metal ion and functional ligand to guide their syntheses of IIPs. ${ }^{17-20}$

With respect to the SPE of $\mathrm{Th}^{4+}$, IIPs exhibit good performances. Buyuktiryaki et al..$^{21}$ prepared a kind of $\mathrm{Th}^{4+}$-IIP beads with $N$-methacryloyl-(L)-glutamic acid as functional ligand to selectively separate $\mathrm{Th}^{4+}$ from $\mathrm{UO}_{2}{ }^{2+}, \mathrm{La}^{3+}$, and $\mathrm{Ce}^{3+}$. Lin and coworkers ${ }^{22}$ synthesized $\mathrm{Th}^{4+}$-IIPs on the surface of silica gel by using 1-phenyl-3-methylthio-4-cyano-5-acrylicacidcarbamoylpyrazole as functional ligand, which was used to pre-concentrate $\mathrm{Th}^{4+}$ before the analysis with UV-vis spectro-photometry. In the syntheses of $\mathrm{Th}^{4+}$-IIPs, many functional ligands were used, which is summarized in Table 1 . It can be found that most of the monomers own carboxyl or amino groups, leading to the optimum $\mathrm{pH}$ value of the IIPs between 3 and 5 . When the $\mathrm{pH}$ value was lower than 3 , the adsorption capacities of these IIPs decreased dramatically. For example, the magnetic $\mathrm{Th}^{4+}$-imprinted chitosan resin prepared by Huang and his coworkers had a large adsorption capacity of $147.1 \mathrm{mg} \mathrm{g}^{-1}$ in a solution of $\mathrm{pH}=$ 4 , but the adsorption capacity was no more than $10 \%$ of the maximum value at the $\mathrm{pH}$ value of $1 .{ }^{23}$ However, in some practical cases, it is necessary to extract $\mathrm{Th}^{4+}$ in high acidity environment. ${ }^{7}$ Recently, this problem began to attract attention in the field of ion-imprinting technology. Fasihi et al. ${ }^{24}$ used vinyl sulfonate, a strong ion-exchanger functional ligand, to prepare $\mathrm{Th}^{4+}-\mathrm{IIP}$, which could start to extract $\mathrm{Th}^{4+}$ with an extraction efficiency of $41 \%$ at $\mathrm{pH}=1.6$. By far, none of these $\mathrm{Th}^{4+}$-IIPs could be used at $\mathrm{pH}<1$. Therefore, there still remains a great challenge. 
Table 1 Summary of the adsorption properties of $\mathrm{Th}^{4+}$-IIPs prepared using different functional ligands in the literature

\begin{tabular}{lll}
\hline Functional ligand & pH range & $\begin{array}{l}\text { Maximum adsorption } \\
\text { capacity/mg g }\end{array}$ \\
\hline$N$-Methacryloyl-(L)-glutamic acid & $2-4$ & $40.44(\mathrm{pH}=3)$ \\
Chitosan-phthalate & $2-4$ & $61.3(\mathrm{pH}=3)$ \\
$N$ - $($-Carboxyphenyl)maleamic acid & $2-5$ & $35.9(\mathrm{pH}=3)$ \\
1-Phenyl-3-methylthio-4-cyano-5-acrylicacidcarbamoyl-pyrazole & $1.5-5$ & $64.8(\mathrm{pH}=3.5)$ \\
Methacrylic acid & $2-5$ & $33.2(\mathrm{pH}=3)$ \\
3-Methyl-1-phenyl-4-(cis-acylbutenoic acid)-2-pyrazolin-5-one & $2-6$ & $56.8(\mathrm{pH}=4.5)$ \\
$N, N^{\prime}$-Bis(3-allyl salicylidene) 0 -phenylenediamine & $3-6$ & $42.54(\mathrm{pH}=4.5)$ \\
Thiosemicarbazide, 4-vinyl pyridine & $2-5$ & $67.28(\mathrm{pH}=4)$ \\
Vinyl sulfonate & $1.5-3.5$ & $9.28(\mathrm{pH}=3)$ \\
Chitosan & $1-6$ & $147.1(\mathrm{pH}=4)$ \\
& & 22 \\
\end{tabular}

Acidic organophosphorus compounds, particularly organophosphorus monacids (such as bis(2-ethylhexyl)-phosphoric acid (HDEHP) ${ }^{10}$ and 2-ethylhexyl phosphoric acid mono-2ethylhexyl ester ${ }^{30}$ ), are widely used in the extraction of $\mathrm{Th}^{4+}$ and trivalent rare earths. Especially, they can extract $\mathrm{Th}^{4+}$ from $1 \mathrm{~mol} \mathrm{~L}^{-1} \mathrm{H}_{2} \mathrm{SO}_{4}$ solution. ${ }^{31}$ Our research group has ever used HDEHP to extract $\mathrm{Th}^{4+}$ by reversed micelles with high efficiency. ${ }^{32,33}$ Dioleylphosphoric acid ${ }^{34,35}$ and 1,12-dodecanediol$O, O^{\prime}$-diphenyl phosphonic acid ${ }^{35}$ were used as functional ligand to synthesize $\mathrm{Zn}^{2+}$-IIP, $\mathrm{La}^{3+}$-IIP, $\mathrm{Ce}^{3+}-$ IIP, and $\mathrm{Dy}^{3+}-$ IIP. Meanwhile, the Dy ${ }^{3+}$-IIP could be used at $\mathrm{pH}=1 .^{34}$ However, to the best of our knowledge, acidic organo-phosphorus compounds have not been used in the preparation of $\mathrm{Th}^{4+}$-IIP. Moreover, with respect to organophosphorus monacids, their coordination behaviors with $\mathrm{Th}^{4+}$ are not well understood. In the literature, there appeared several complex compounds with different molar ratios of ligand to $\mathrm{Th}^{4+}$, e.g. $\mathrm{Th}\left(\mathrm{NO}_{3}\right)(\mathrm{DEHP})_{3},{ }^{32}$ $\mathrm{Th}\left(\mathrm{NO}_{3}\right)_{2}(\mathrm{DEHP})_{2},{ }^{33} \mathrm{Th}\left[\mathrm{H}(\mathrm{DEHP})_{2}\right]_{4},{ }^{36}$ and $\mathrm{Th}\left(\mathrm{NO}_{3}\right)_{2}(\mathrm{DcyHPA})_{2}$ (DcyHPA: dicyclohexylphosphinic acid). ${ }^{37}$ Therefore, it is essential to determine the molar ratio of functional ligand to $\mathrm{Th}^{4+}$ when organophosphorus monacid is applied as functional ligand to synthesize $\mathrm{Th}^{4}$-IIP.

Since organophosphorus monacids have strong affinities towards $\mathrm{Th}^{4+}, \mathrm{Th}^{4+}$-IIP was synthesized in this work by using bis(2-methacryloxyethyl) phosphate (BMAOP) as the functional ligand. Also, the molar ratio of functional ligand to $\mathrm{Th}^{4+}$ was optimized in detail.

\section{Experimental}

\subsection{Reagents and instruments}

Bis(2-methacryloxyethyl) phosphate (BMAOP, N/A, J\&K Scientific, China), ethylene glycol dimethacrylate (EGDMA, 98\%, Acros, Belgium) and dimethyl phosphate (DMP, 98\%, Acros, Belgium) were used as received. Azobisisobutyronitrile (AIBN, A.R., Beijing Yili Fine Chemical Products Inc., China) was purified by recrystallization. Ultrapure water was used throughout the experiments. All other chemical reagents used in this study were of analytical grade and used without further purification.

The concentrations of all the metal ions were determined by inductively coupled plasma-atomic emission spectrometer (ICP-
AES, Leeman, USA) with relative standard deviation (RSD) below $5 \%$. Thermo-gravimetric analysis (TGA) was carried out using a TGA-DSC-DTA (Q-600 SDT, Thermal Analysis Co., USA). The Brunauer-Emmett-Teller (BET) surface area was measured using an accelerated surface area \& porosimetry system (ASAP 2010, Micrometer, USA). FT-IR spectra were recorded in the frequency range of $400-4000 \mathrm{~cm}^{-1}$ using FT-IR spectrometer (Tensor 27 , Bruker, Germany). ${ }^{31} \mathrm{P}$ NMR spectra were obtained with phosphoric acid as external standard by using Bruker- $400 \mathrm{MHz}$ NMR (ARX400, Bruker, Switzerland). The elemental analysis was taken by using an Elemental Analyzer (Vario EL, Elementar Analysensysteme GmbH, Germany). A pH meter (Delta 320, MettlerToledo, Switzerland) was used to measure $\mathrm{pH}$ values.

Sample for Extended X-ray Absorption Fine Structure (EXAFS) measurement was prepared by mixing $\mathrm{Th}\left(\mathrm{NO}_{3}\right)_{4} \cdot 4 \mathrm{H}_{2} \mathrm{O}$, DMP and triethylamine $\left(\mathrm{Et}_{3} \mathrm{~N}\right)$ at a molar ratio of $1: 4: 4$ in dimethyl sulphoxide (DMSO) in a $2 \mathrm{~mL}$ plastic tube, and then measured directly. XAFS measurement at Th L3-edge in transmission mode was performed at the BL14W1 (ref. 38) in Shanghai Synchrotron Radiation Facility (SSRF). The electron beam energy was $3.5 \mathrm{GeV}$ and the stored current was $260 \mathrm{~mA}$ (top-up). A 38-pole wiggler with the maximum magnetic field of 1.2 $\mathrm{T}$ inserted in the straight section of the storage ring was used. XAFS data were collected using a fixed-exit double-crystal Si (111) monochromator. The energy was calibrated using $\mathrm{Zr}$ foil. The photon flux at the sample position was $6.9 \times 10^{11}$ photons per second. The raw data analysis was performed using IFEFFIT software package according to the standard data analysis procedures..$^{39}$ The spectrum was calibrated, averaged, pre-edge background subtracted, and post-edge normalized using Athena program in IFEFFIT software package. The Fourier transformation of the $k^{3}$-weighted EXAFS oscillations, $k^{3} \times \chi(k)$, from $k$ space to $R$ space was performed over a range of $2.3-10.3 \AA^{-1}$ to obtain a radial distribution function. And data fitting was done by Artemis program in IFEFFIT.

\subsection{Synthesis of $\mathrm{Th}^{4+}$-imprinted polymer}

In a typical preparation process, $0.322 \mathrm{~g}(1.0 \mathrm{mmol})$ BMAOP and $0.101 \mathrm{~g} \mathrm{Et}_{3} \mathrm{~N}(1.0 \mathrm{mmol})$ were dissolved in $2.5 \mathrm{~mL}$ DMSO, and stirred for $0.5 \mathrm{~h}$. Then, $0.138 \mathrm{~g} \mathrm{Th}\left(\mathrm{NO}_{3}\right)_{4} \cdot 4 \mathrm{H}_{2} \mathrm{O}(0.25 \mathrm{mmol})$ was added and stirred for $2 \mathrm{~h}$. After adding $0.792 \mathrm{~g}$ EGDMA ( $4 \mathrm{mmol}$ ) and $10 \mathrm{mg}$ AIBN, the mixture was purged with $\mathrm{N}_{2}$ for $20 \mathrm{~min}$ and 
polymerized in an oil bath at $60{ }^{\circ} \mathrm{C}$ for $18 \mathrm{~h}$. The bulk polymer was grounded and stirred in $100 \mathrm{~mL} 0.1 \mathrm{~mol} \mathrm{~L}^{-1}$ ethylenediaminetetraacetic acid disodium salt $\left(\mathrm{Na}_{2}\right.$ EDTA) for several times to remove $\mathrm{Th}^{4+}$. Then, the polymer was washed with water and acetone in turn. At last, the dried polymer was sieved to get the particles between 80 and 200 mesh. The non-imprinted polymer (NIP) was prepared under similar conditions except the absence of $\mathrm{Th}\left(\mathrm{NO}_{3}\right)_{4} \cdot 4 \mathrm{H}_{2} \mathrm{O}$ and $\mathrm{Et}_{3} \mathrm{~N}$.

\subsection{Adsorption experiments}

The adsorption capacities of IIP (NIP) were determined by stirring $0.0050 \mathrm{~g}$ IIP (NIP) with a $0.0100 \mathrm{~L}$ solution of $\mathrm{Th}\left(\mathrm{NO}_{3}\right)_{4}$ $\left(15-60 \mathrm{mg} \mathrm{L}^{-1}\right)$ and $\mathrm{HCl}\left(10^{-3.8}-6 \mathrm{~mol} \mathrm{~L}^{-1}\right)$ at $25{ }^{\circ} \mathrm{C}$. Then, the mixtures were centrifuged at $4000 \mathrm{rpm}$ for $5 \mathrm{~min}$, and the concentration of $\mathrm{Th}^{4+}$ in the solutions was measured by ICPAES. The adsorption capacity $\left(Q, \mathrm{mg}^{-1}\right)$ and distribution ratio $\left(k_{\mathrm{d}}, \mathrm{mL} \mathrm{g}^{-1}\right)$ was calculated according to eqn (1) and (2).

$$
\begin{gathered}
Q=\left(C_{0}-C_{\mathrm{e}}\right) V / W \\
k_{\mathrm{d}}=\left[\left(C_{0}-C_{\mathrm{e}}\right) V / C_{\mathrm{e}} W\right] \times 1000
\end{gathered}
$$

where $C_{0}$ and $C_{\mathrm{e}}\left(\mathrm{mg} \mathrm{L}^{-1}\right)$ are the concentrations of the metal ions before and after adsorption, respectively, while $V(\mathrm{~L})$ is the volume of the testing solution and $W(\mathrm{~g})$ the weight of adsorbent.

In the selectivity studies, $0.0100 \mathrm{~g}$ of the IIP (NIP) was shaken with a solution containing both $\mathrm{Th}^{4+}$ and the other metal ion, where the concentration of each metal ion was $20 \mathrm{mg} \mathrm{L}^{-1}$. The selectivity coefficient $S$ and the relative selectivity coefficient $k^{\prime}$ were calculated according to eqn (3) and (4).

$$
\begin{gathered}
S_{\mathrm{Th} / \mathrm{M}}=k_{\mathrm{d}(\mathrm{Th})} / k_{\mathrm{d}(\mathrm{M})} \\
k^{\prime}=S_{\mathrm{IIP}} / S_{\mathrm{NIP}}
\end{gathered}
$$

\section{Results and discussion}

\subsection{Optimizing the molar ratio of functional ligand to $\mathrm{Th}^{4+}$}

Before the syntheses of the $\mathrm{Th}^{4+}$-IIP, the molar ratio of the functional ligand to $\mathrm{Th}^{4+}$ should be determined. The functional ligand BMAOP is not stable at room temperature and easy to polymerize. Thus, DMP was chosen as a stable substitute for BMAOP, because each of them has one phosphoric acid group $\left({ }_{0}-{ }_{0} \leqslant_{0}^{O H}\right)$ to coordinate with metal ions.

Firstly, ${ }^{31} \mathrm{P}$ NMR titration was used. With the change of the concentration of DMP (deprotonated with the same amount of $\left.\mathrm{Et}_{3} \mathrm{~N}\right)$, the ${ }^{31} \mathrm{P}$ NMR spectra were measured at the $\mathrm{Th}\left(\mathrm{NO}_{3}\right)_{4}$ concentration of $0.25 \mathrm{mmol} \mathrm{L}^{-1}$. As can be seen from Fig. 1, the peak of the free ligand around $0.8 \mathrm{ppm}$ starts to appear when the molar ratio of DMP to $\mathrm{Th}^{4+}$ arrives at 3.9 and the intensity of the peak grows fast beyond 4, suggesting the formation of a relatively stable $4: 1$ complex compound between DMP and $\mathrm{Th}^{4+}$.

Moreover, the complex compound of DMP with $\mathrm{Th}^{4+}$ was synthesized to confirm the molar ratio of DMP to $\mathrm{Th}^{4+}$. After mixing $\mathrm{Th}\left(\mathrm{NO}_{3}\right)_{4}, \mathrm{DMP}$ and $\mathrm{Et}_{3} \mathrm{~N}$ at a molar ratio of $1: 4: 4$ in

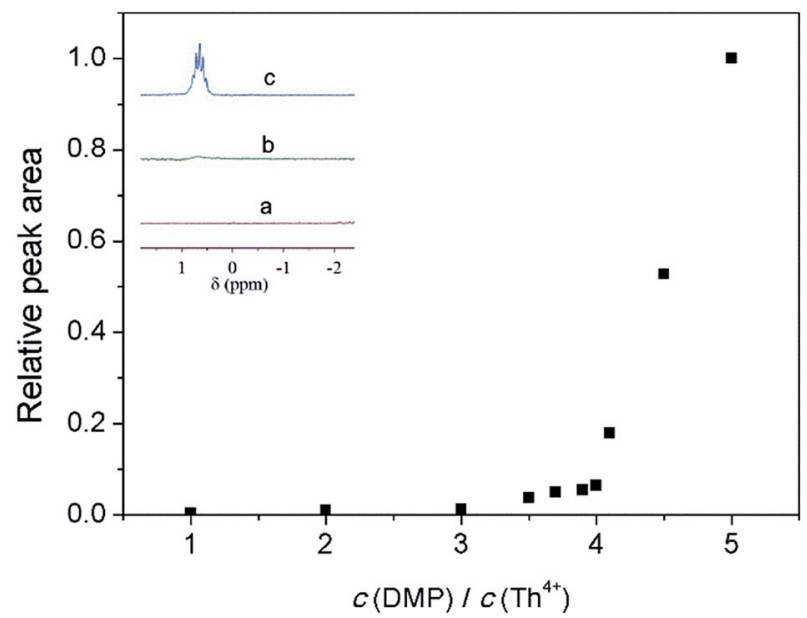

Fig. $1{ }^{31} \mathrm{P}$ NMR study of the $\mathrm{Th}^{4+}-\mathrm{DMP}$ complex compound. The relationship between the relative peak area of free ligand and $n$ (DMP)/ $n\left(\mathrm{Th}^{4+}\right)$. (The peak area of free ligand is defined as 1.0 at the $n$ (DMP)/ $n\left(\mathrm{Th}^{4+}\right)$ value of 5). Inset: partial ${ }^{31} \mathrm{P}$ NMR spectra of the solution with different $n(\mathrm{DMP}) / n\left(\mathrm{Th}^{4+}\right)$ : 1 (a), 3.9 (b), 4.5 (c).

DMSO, water was added. Then, white precipitates were collected and dried in vacuum. Elemental analysis showed that the composition of the compound is close to that of Th(DMP) $)_{4} \cdot \mathrm{H}_{2} \mathrm{O}$ (elemental analysis (\%) calculated for $\mathrm{Th}\left(\mathrm{C}_{2} \mathrm{H}_{6} \mathrm{O}_{4} \mathrm{P}\right)_{4} \cdot \mathrm{H}_{2} \mathrm{O}$ : C 12.81, $\mathrm{H}$ 3.49; found: $\left.\mathrm{C} 12.91, \mathrm{H} 3.34\right)$.

Furthermore, EXAFS was used to determine the coordination number of $\mathrm{Th}^{4+}$ when the molar ration of $\mathrm{DMP}$ to $\mathrm{Th}^{4+}$ is 4 . The fitting curve of the EXAFS data according to the single scattering theoretical phase and amplitude functions is shown in Fig. 2. The result indicates that a shell of $9.1 \pm 0.7$ oxygen atoms directly coordinated to $\mathrm{Th}^{4+}$ at approximately $2.39 \pm 0.01 \AA$ (Table 2). Since organophosphorus monacids often coordinate with $\mathrm{Th}^{4+}$ as bidentate ligands, ${ }^{37}$ combining the results of ${ }^{31} \mathrm{P}$ NMR and elemental analysis, it is reasonable to conclude that four bidentate DMPs and one monodentate ligand (water or DMSO) coordinate with $\mathrm{Th}^{4+}$ in the solution (Scheme 1).

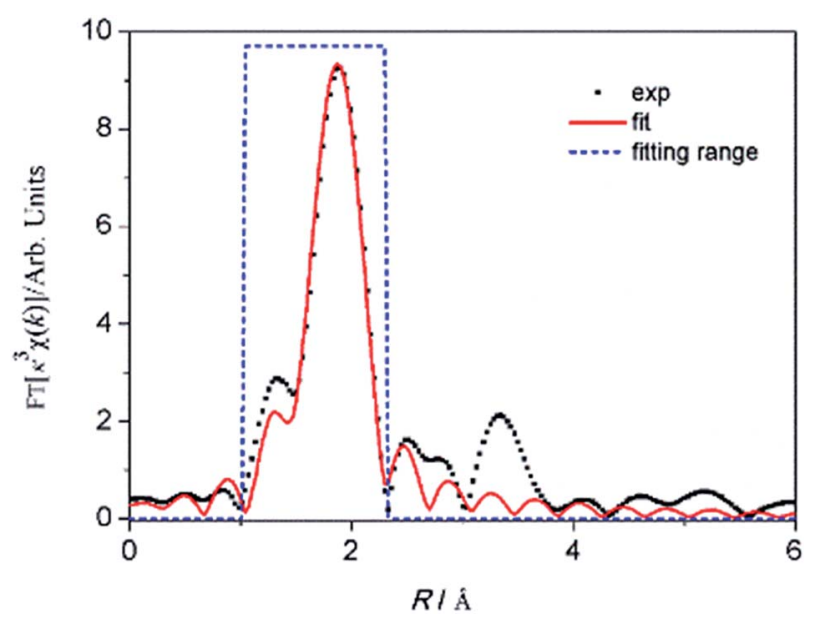

Fig. 2 The $k^{3}$-weighted EXAFS Fourier transform spectrum (dots) and the best fitting (line) of $\mathrm{Th}^{4+}$ in the solution when $c(\mathrm{DMP}) / c\left(\mathrm{Th}^{4+}\right)$ is 4 . 
Table 2 Results of respective coordination number $(\mathrm{CN})$ and $\mathrm{Th}^{4+}-\mathrm{O}$ bond length $(R)$ by fitting two shells of the $k^{3}$ weighted Th $L 3$ edge EXAFS

\begin{tabular}{|c|c|c|c|c|c|}
\hline & $\mathrm{CN}$ & $R / \AA ̊$ & $\sigma^{2} / 10^{-3} \AA^{2}$ & $\Delta E_{0} / \mathrm{eV}$ & $R$ factor \\
\hline Th-O & $9.1 \pm 0.7$ & $2.39 \pm 0.01$ & $5.2 \pm 0.3$ & $4.4 \pm 1.0$ & 0.004 \\
\hline
\end{tabular}

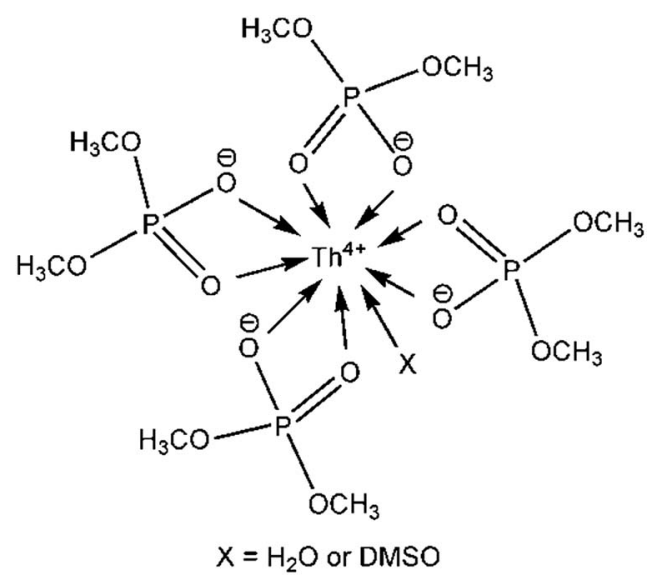

Scheme 1 Scheme of the complex compound of DMP and $\mathrm{Th}^{4+}$.
Then, fixing the molar ratio of EGDMA to BMAOP at 4, IIP1IIP4, with the molar ratios of BMAOP to $\mathrm{Th}^{4+}$ at 3, 3.5, 4 and 5 respectively, were synthesized. Meanwhile, the synthesis process of IIP3 is exhibited in Scheme 2. As can be seen from Fig. 3, the adsorption capacity of IIP3 is the highest. However, NIP shows high adsorption capacity, too, which is slightly higher than that of IIP1. In general, imprinting effect or large surface area always leads to a high adsorption capacity. Herein, the surface areas of IIP1-4 and NIP were determined to be 164, 182, 217, 210, and $196 \mathrm{~m}^{2} \mathrm{~g}^{-1}$, respectively. For the larger surface area, the adsorption capacity of NIP is slightly higher than that of IIP1. As the imprinting effect, the adsorption capacity of IIP2 is higher than that of NIP although the surface area of IIP2 is slightly lower than that of NIP. The reasons for the large surface area and high adsorption capacity of NIP will be discussed in detail in Sections 3.2.3 and 3.3.3, respectively. We also digested the IIPs, and found that the residual thorium was less than $3 \mathrm{mg} \mathrm{g}^{-1}$. In other words, the residual thorium in the IIPs has little influence on the adsorption capacities.

Furthermore, the selectivity coefficient $\left(\mathrm{Th}^{4+} / \mathrm{Eu}^{3+}\right)$ of IIP3 are obviously higher than those of IIP1, IIP2, IIP4, and much higher than that of NIP (Fig. 3). This could be ascribed to the fact that one $\mathrm{Th}^{4+}$ ion coordinates with four BMAOP molecules, and excessive or insufficient functional ligands may reduce the

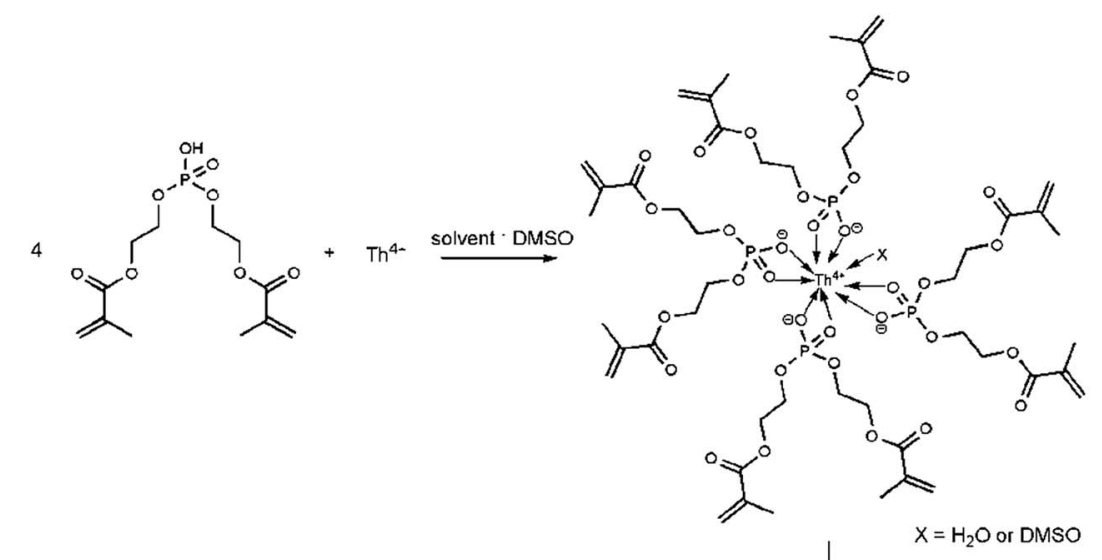

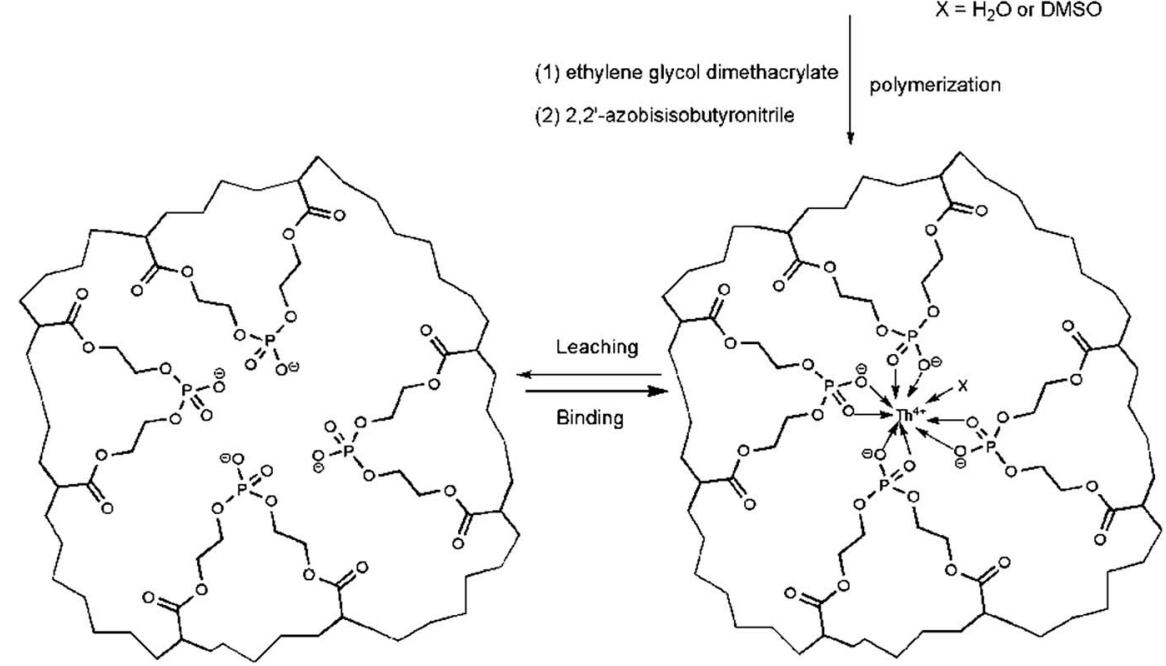

Scheme 2 Scheme for the preparation of $\mathrm{Th}^{4+}-$ IIP3. 


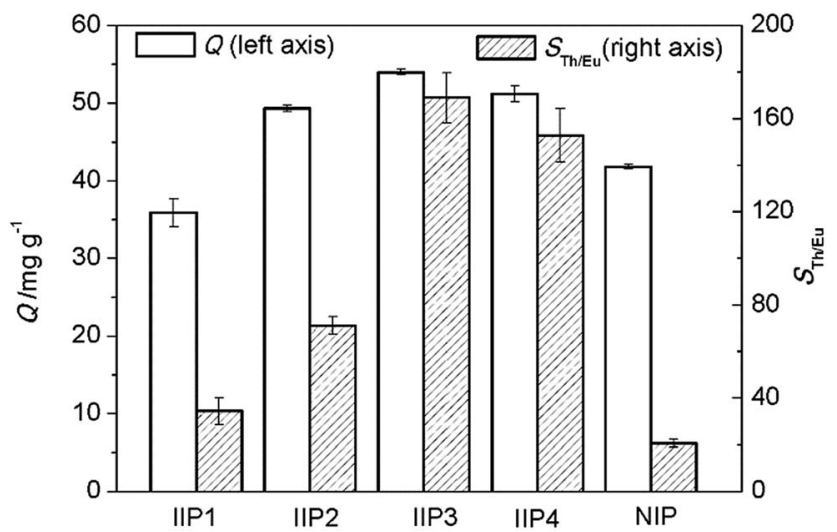

Fig. 3 Adsorption capacities and selectivity coefficients $\left(\mathrm{Th}^{4+} / \mathrm{Eu}^{3+}\right)$ of IIPs with different ratios of BMAOP to $\mathrm{Th}^{4+}$ (from IIP1 to IIP4, $n(\mathrm{BMAOP}) / n\left(\mathrm{Th}^{4+}\right)=3,3.5,4$, and 5) and NIP. (Adsorbent: $0.0050 \mathrm{~g}$, metal ions concentration: $30 \mathrm{mg} \mathrm{L}^{-1}$, solution: $0.0100 \mathrm{~L}, \mathrm{pH}$ : 3.0, time: $12 \mathrm{~h})$.

specificity of the binding sites. The proper molar ratio of BMAOP to $\mathrm{Th}^{4+}$ should be $4: 1$, consistent with the results of ${ }^{31} \mathrm{P}$ NMR, elemental analysis and EXAFS. Thus, IIP3 was selected in the following characterization and adsorption experiments.

\subsection{Characterization}

3.2.1 FT-IR spectra. The FT-IR spectra of the IIP3 and NIP (before and after washing with $\mathrm{Na}_{2}$ EDTA solution) show similar backbones because of their identical components (Fig. 4). All of the spectra show a strong absorption peak at $1725 \mathrm{~cm}^{-1}$ attributed to the stretching vibration of $\mathrm{C}=\mathrm{O}$ group and a peak at $1160 \mathrm{~cm}^{-1}$ assigned to the stretching vibration of $\mathrm{P}=\mathrm{O}$ group. These peaks demonstrate that BMAOP has been successfully copolymerized with EGDMA, and IIP3 has an identical composition as NIP. After washing, the absorption at $1022 \mathrm{~cm}^{-1}$, the stretching vibration of $\mathrm{S}=\mathrm{O}$ group, diminished, indicating the leaching of the residual DMSO.

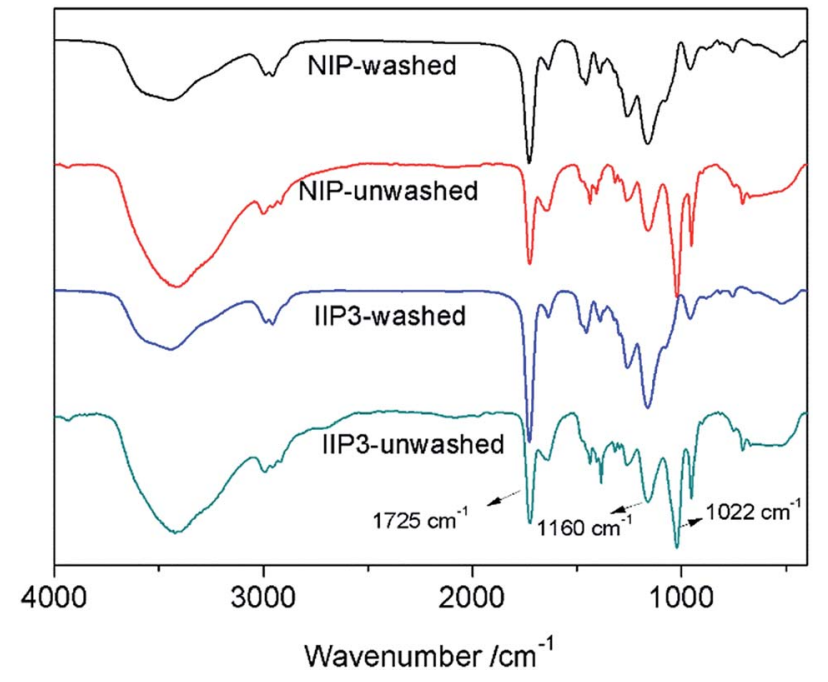

Fig. 4 FT-IR spectra of (un)washed IIP3 and NIP.
3.2.2 Thermal analysis (TGA). Thermal stabilities of the IIP3 and NIP particles were investigated by thermo-gravimetric analyses (Fig. 5). In the measurement process, the samples were heated from room temperature to $600{ }^{\circ} \mathrm{C}$ with a heating rate of $10{ }^{\circ} \mathrm{C} \mathrm{min}{ }^{-1}$ in air atmosphere. The TGA plots of IIP3 and NIP are very similar, indicating their identical components. Since the thermal decomposition temperatures of IIP3 and NIP are up to $240^{\circ} \mathrm{C}$, they can be used stably in the common environments.

3.2.3 Surface area analysis. The surface area is an important parameter for adsorption materials which affect the adsorption kinetics and capacity greatly. Generally, it is believed that materials with larger surface area have a higher adsorption capacity. ${ }^{40}$ Herein, the BET surface areas of the IIP3 and NIP were 217 and $196 \mathrm{~m}^{2} \mathrm{~g}^{-1}$, respectively, and they had similar pore size (IIP3: $0.46 \mathrm{~nm}$, NIP: $0.49 \mathrm{~nm}$ ) and pore volume (IIP3: 8.4 $\mathrm{cm}^{3} \mathrm{~g}^{-1}$, NIP: $9.9 \mathrm{~cm}^{3} \mathrm{~g}^{-1}$ ). The large surface areas of IIP3 and NIP may be ascribed to the presence of DMSO in the preparation process. The functional ligand (BMAOP) has a similar methacryloxyethyl part as the cross-linker (EGDMA), leading to a similar polymerization ability. Since DMSO has a good thermodynamic compatibility for poly(EGDMA), the phase separation will be late in the polymerization, resulting in IIP3 (NIP) with large surface area. ${ }^{41}$

\subsection{Adsorption properties}

3.3.1 Effect of the acidity. The effect of acidity was investigated by altering the concentration of $\mathrm{HCl}$ from $10^{-3.8}$ $(\mathrm{pH}=3.8)$ to $6.0 \mathrm{~mol} \mathrm{~L}^{-1}$ (Fig. 6). With the increase of the $\mathrm{HCl}$ concentration, the adsorption capacity of IIP3 decreases, while that of NIP increases to a maximum value at $\mathrm{pH}=2$ and then decreases. Moreover, the IIP3 exhibits a greater retention capacity than NIP at $\mathrm{pH}<1$ and $\mathrm{pH}>2$, while their retention capacities are almost identical at the $\mathrm{pH}$ range of $1-2$. This interesting phenomenon may be attributed to the competition adsorption. In acidic solution, $\mathrm{H}^{+}$competes with $\mathrm{Th}^{4+}$ for combining the phosphoric acid group on the polymer, which becomes much serious at $\mathrm{pH}<1$. When the $\mathrm{pH}$ value is higher than 2 , the $\mathrm{Th}^{4+}$ starts to hydrolyze, and $\mathrm{OH}^{-}$competes with the

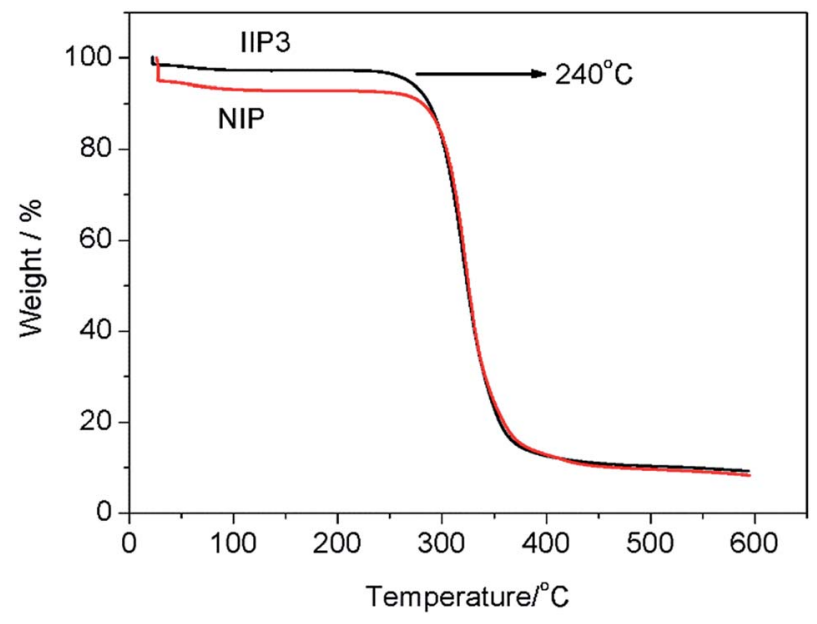

Fig. 5 TGA plots of IIP3 and NIP particles. 


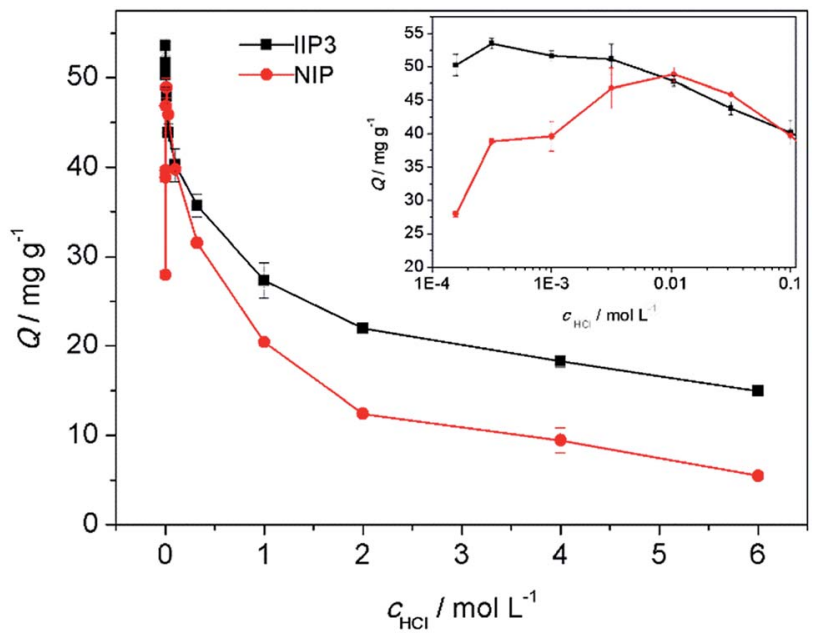

Fig. 6 Effect of acidity on the adsorption capacities of IIP3 and NIP. (Adsorbent: $0.0050 \mathrm{~g}$, thorium concentration: $30 \mathrm{mg} \mathrm{L}^{-1}$, thorium solution: $0.0100 \mathrm{~L}$, time: $12 \mathrm{~h}$ ).

phosphoric acid group in the binding of $\mathrm{Th}^{4+}$. These two competitions lead to the fact that the adsorption capacity of NIP reaches its maximum value at $\mathrm{pH}=2$. While, in the intense competition situation, the specific binding sites in the IIP3 can combine with $\mathrm{Th}^{4+}$ more effectively than the randomly distributed functional groups in the NIP, leading to a larger adsorption capacity at $\mathrm{pH}<1$ and $\mathrm{pH}>2$. The IIP3 has a maximum adsorption capacity of $53.5 \mathrm{mg} \mathrm{g}^{-1}$ at $\mathrm{pH}=3.5$, which is comparable with the theoretical capacity $\left(52.1 \mathrm{mg} \mathrm{g}^{-1}\right)$ estimated according to the feed ratio. Different from the $\mathrm{Th}^{4+}$-IIPs reported in the literature which hardly adsorb $\mathrm{Th}^{4+}$ at $\mathrm{pH}=1$ (Table 1), the $\mathrm{Th}^{4+}$-IIP3 has $50 \%$ of the maximum adsorption capacity in $1.0 \mathrm{~mol} \mathrm{~L}^{-1} \mathrm{HCl}$ and still has $25 \%$ of the maximum adsorption capacity even in $6.0 \mathrm{~mol} \mathrm{~L}^{-1} \mathrm{HCl}$. It may be attributed to the low $\mathrm{p} K_{\mathrm{a}}$ value of the functional ligand, and the strong coordination interaction between $\mathrm{Th}^{4+}$ and phosphoric acid group. To investigate the adsorption ability of the $\mathrm{Th}^{4+}$ IIP3 in high acidity environment, the following experiments were performed in $1.0 \mathrm{~mol} \mathrm{~L}^{-1} \mathrm{HCl}$.

3.3.2 Adsorption kinetics. According to the effect of time on the adsorption of $\mathrm{Th}^{4+}$ from aqueous solutions (Fig. 7), the equilibrium times of IIP3 and NIP were determined to be $c a .8 \mathrm{~h}$. To guarantee adsorption equilibrium, the adsorption time was chosen to be $12 \mathrm{~h}$ in the following studies.

To explore the mechanism of adsorption kinetics, two different kinetic models were applied to fit the experimental data, i.e., the pseudo-first-order kinetic model (eqn (5)) and the pseudo-second-order kinetic model (eqn (6)).

$$
\begin{aligned}
Q_{t} & =Q_{\mathrm{e}}\left(1-\mathrm{e}^{-k_{1} t}\right) \\
Q_{t} & =t /\left(\frac{t}{Q_{\mathrm{e}}}+\frac{Q_{\mathrm{e}}{ }^{2}}{k_{2}}\right)
\end{aligned}
$$

where $Q_{\mathrm{e}}\left(\mathrm{mg} \mathrm{g}^{-1}\right)$ and $Q_{t}\left(\mathrm{mg} \mathrm{g}^{-1}\right)$ are the adsorption capacities at equilibrium and at time $t(\mathrm{~h})$, respectively; $k_{1}\left(\mathrm{~h}^{-1}\right)$ and $k_{2}(\mathrm{mg}$ $\left.\mathrm{g}^{-1} \mathrm{~h}^{-1}\right)$ are the adsorption rate constants related to pseudo-

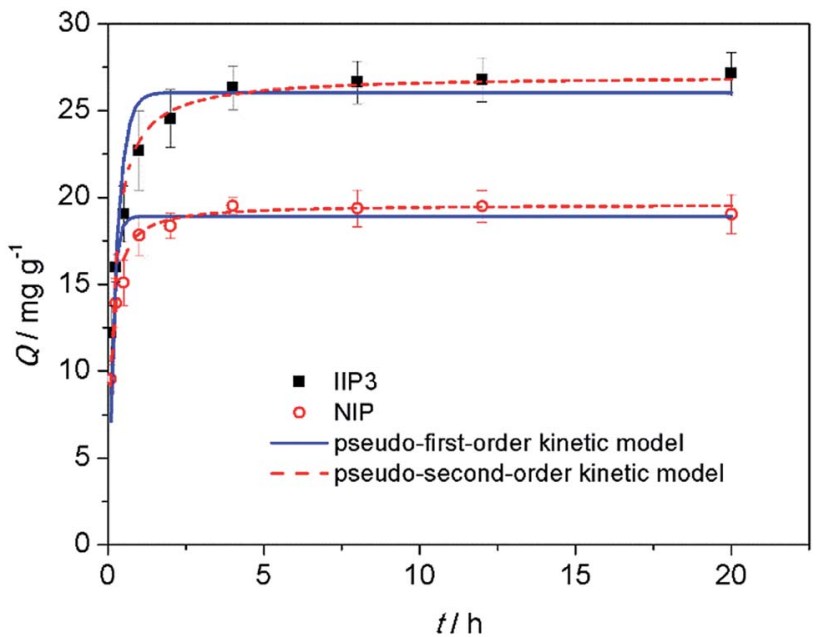

Fig. 7 Effect of time on the adsorption of $\mathrm{Th}^{4+}$ on the IIP3 and NIP. (Adsorbent: $0.0050 \mathrm{~g}$, thorium concentration: $20 \mathrm{mg} \mathrm{L}^{-1}$, thorium solution: $0.0100 \mathrm{~L}, \mathrm{HCl}: 1.0 \mathrm{~mol} \mathrm{~L}^{-1}$ ).

first-order and pseudo-second-order kinetic models, respectively. The pseudo-first-order kinetic model assumes that the rate of adsorption site occupation is proportional to the number of unoccupied sites, while the pseudo-second-order kinetic model corresponds to the chemical reaction mechanisms, in which the adsorption rate are controlled by chemical adsorption via sharing or exchange of electrons between the adsorbate and adsorbent. ${ }^{42}$ In this study, the pseudo-second-order kinetic model is much better than the pseudo-first-order kinetic model in the fitting of the experimental data (Fig. 7 and Table 3), suggesting that the adsorption is a chemical coordination process.

3.3.3 Adsorption isotherm. The adsorption capacities of IIP3 and NIP increased with the increase of equilibrium concentrations (Fig. 8). The isothermal adsorption data were analyzed with the Langmuir (eqn (7)) and Freundlich (eqn (8)) equations.

$$
\begin{gathered}
Q=Q_{\mathrm{m}} b C_{\mathrm{e}} /\left(1+C_{\mathrm{e}}\right) \\
Q=K_{\mathrm{F}} C_{\mathrm{e}^{n}}^{\frac{1}{n}}
\end{gathered}
$$

where $C_{\mathrm{e}}\left(\mathrm{mg} \mathrm{L}^{-1}\right)$ is the equilibrium concentration of $\mathrm{Th}^{4+}, Q_{\mathrm{m}}$ $\left(\mathrm{mg} \mathrm{g}^{-1}\right)$ is the maximum adsorption quantity, $b\left(\mathrm{~L} \mathrm{mg}^{-1}\right)$ is the Langmuir constant, $K_{\mathrm{F}}$ and $n$ are the Freundlich constants. It can be seen from Table 4 and Fig. 8 that Langmuir model is more suitable in the fitting of the isothermal adsorption data of IIP3, while Freundlich model fits those of NIP better. The maximum adsorption capacity of IIP3 calculated from the Langmuir isotherm was $33.3 \mathrm{mg} \mathrm{g}^{-1}$.

There are specific binding sites and unspecific binding sites in IIPs, while the binding sites in NIP is unspecific. The surface area of NIP is slightly lower than that of IIP3, suggesting that the accessibility of the binding sites of IIP3 and NIP is similar.

When the concentration of $\mathrm{Th}^{4+}$ is low, the specific binding sites in IIP3 show stronger binding ability than the unspecific binding sites in NIP. For example, at an initial $\mathrm{Th}^{4+}$ 
Table 3 Comparison of the fitting results of the pseudo-first-order and pseudo-second-order kinetic models

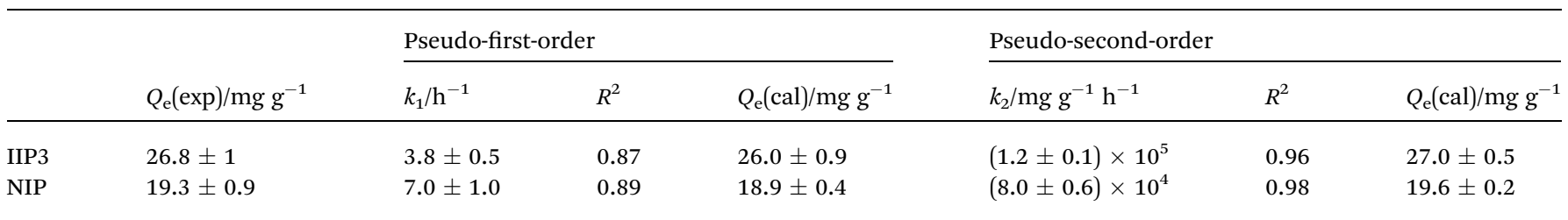

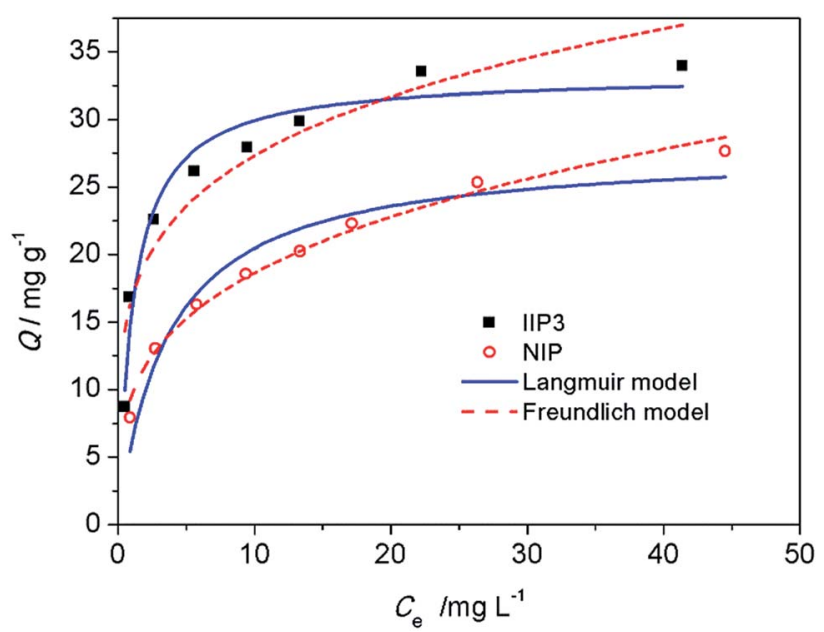

Fig. 8 Adsorption isotherms of IIP3 and NIP. (Adsorbent: $0.0050 \mathrm{~g}$, thorium solution: $0.0100 \mathrm{~L}, \mathrm{HCl}: 1.0 \mathrm{~mol} \mathrm{~L}^{-1}$, time: $12 \mathrm{~h}$ ).

Table 4 Comparison of the fitting results of the Langmuir and Freundlich models

\begin{tabular}{|c|c|c|c|c|c|c|}
\hline & \multicolumn{3}{|c|}{ Langmuir } & \multicolumn{3}{|c|}{ Freundlich } \\
\hline & $R^{2}$ & $Q_{\mathrm{m}} / \mathrm{mg} \mathrm{g}^{-1}$ & $b / \mathrm{L} \mathrm{mg}^{-1}$ & $R^{2}$ & $K_{\mathrm{F}}$ & $n$ \\
\hline IIP3 & 0.95 & $33.3 \pm 1.0$ & $0.9 \pm 0.2$ & 0.88 & $17 \pm 2$ & $4.7 \pm 0.7$ \\
\hline NIP & 0.93 & $27.8 \pm 2.0$ & $0.28 \pm 0.06$ & 0.99 & $9.6 \pm 0.4$ & $3.5 \pm 0.2$ \\
\hline
\end{tabular}

concentration of $5.2 \mathrm{mg} \mathrm{L}^{-1}$, the $k_{\mathrm{d}}$ values of IIP3 and NIP are $1.7 \times 10^{4}$ and $8.1 \times 10^{3} \mathrm{~mL} \mathrm{~g}^{-1}$, respectively, indicating that the IIP3 can adsorb $\mathrm{Th}^{4+}$ more effectively. When the $\mathrm{Th}^{4+}$ in the solution is abundant, both the specific binding sites and unspecific binding sites in IIP3, as well as the unspecific binding sites in NIP can combine with $\mathrm{Th}^{4+}$. Thus, with the increase of the equilibrium concentration of $\mathrm{Th}^{4+}$, the difference of adsorption capacities between IIP3 and NIP becomes smaller (Fig. 8).

3.3.4 Adsorption selectivity. To investigate the selectivity of the IIP3, competitive adsorptions were performed. In this investigation, $\mathrm{UO}_{2}{ }^{2+}, \mathrm{Fe}^{3+}, \mathrm{La}^{3+}, \mathrm{Eu}^{3+}$, and $\mathrm{Dy}^{3+}$ were chosen as competitive metal ions because they often coexist in minerals as well as in waste water., ${ }^{3,4}$ The obtained $k_{\mathrm{d}}, S$ and $k^{\prime}$ (Table 5) show that IIP3 can effectively extract $\mathrm{Th}^{4+}$ from aqueous solution containing various interfering ions, especially in the presence of lanthanide ions. Also, $0.0100 \mathrm{~g}$ IIP3 (NIP) was used to extract $\mathrm{Th}^{4+}$ from a mixed solution of $\mathrm{Th}^{4+}$ and the above competing ions with each concentration of $20 \mathrm{mg} \mathrm{L}^{-1}$. The result (Fig. 9) indicates that $\mathrm{Th}^{4+}$ can be effectively extracted from the mixture solution, and only a few $\mathrm{UO}_{2}{ }^{2+}$ is co-extracted.

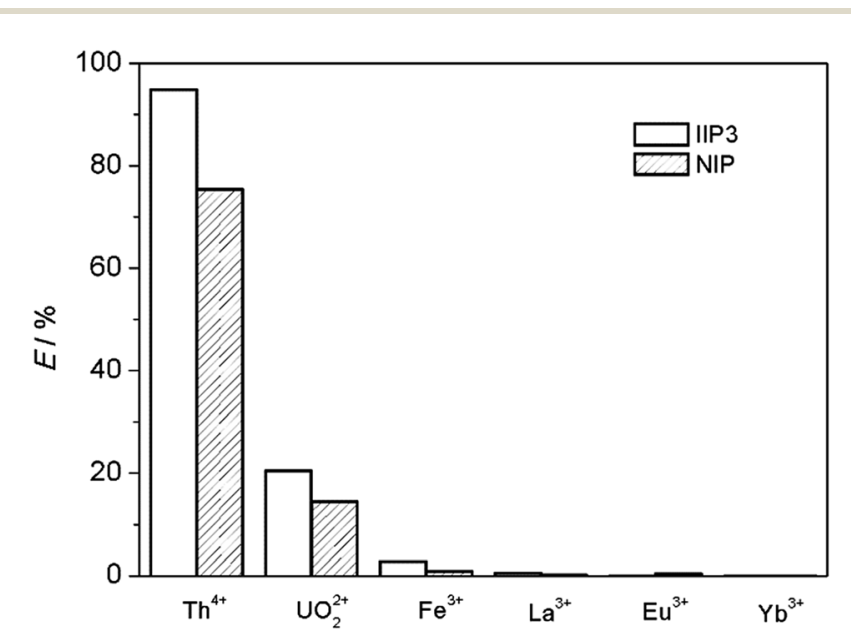

Fig. 9 Extraction of $\mathrm{Th}^{4+}$ and other competing ions from mixed solution. (Adsorbent: $0.0100 \mathrm{~g}$, each metal ion concentration: $20 \mathrm{mg} \mathrm{L}^{-1}$, solution: $0.0100 \mathrm{~L}, \mathrm{HCl}: 1.0 \mathrm{~mol} \mathrm{~L}^{-1}$, time: $12 \mathrm{~h}$ ).

Table 5 Selective adsorption properties of the IIP3 and NIP

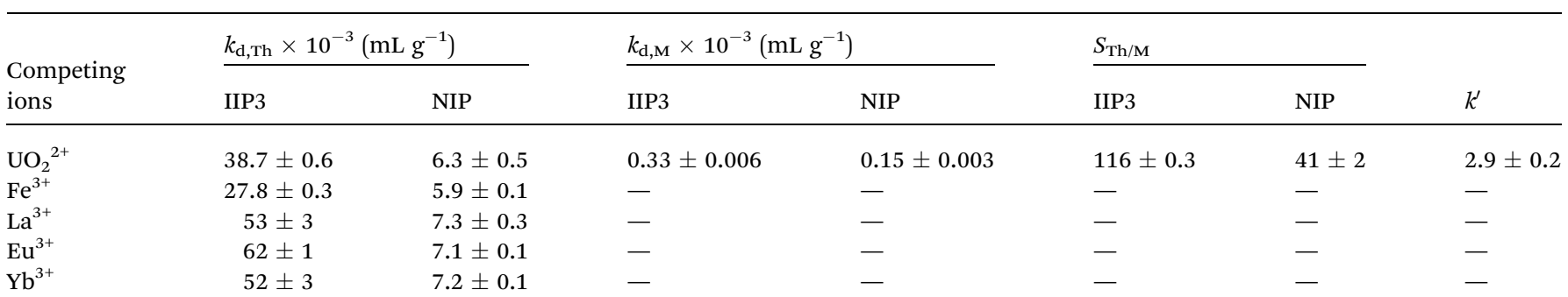

${ }^{a}$ The adsorptions of $\mathrm{Fe}^{3+}$ and $\mathrm{Ln}^{3+}$ on the IIP3 and NIP could not be detected. 


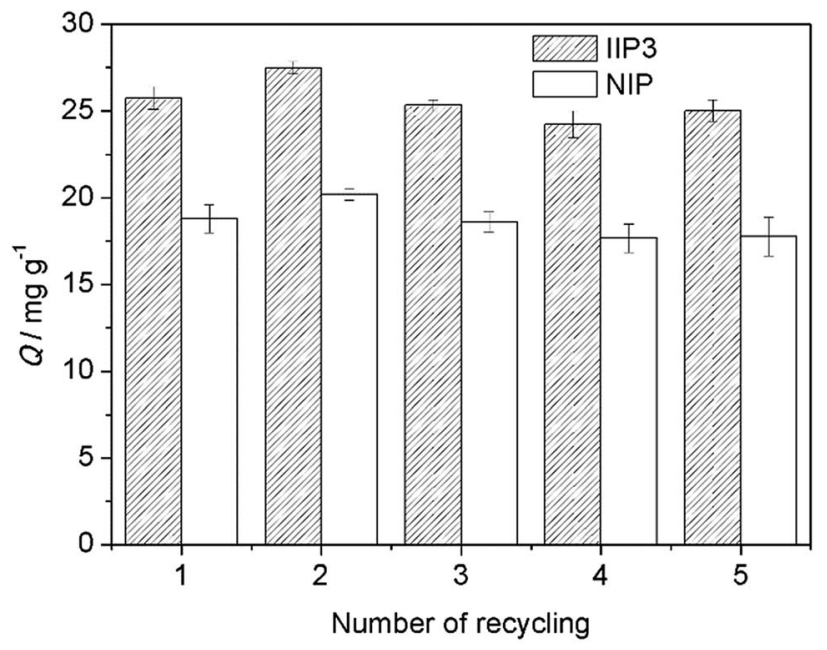

Fig. 10 The retention capacities of the recycled $\mathrm{Th}^{4+}-$ IIP3 and NIP particles in five times. (Adsorbent: $0.0050 \mathrm{~g}$, thorium concentration: $20 \mathrm{mg} \mathrm{L}^{-1}$, thorium solution: $0.0100 \mathrm{~L}, \mathrm{HCl}: 1.0 \mathrm{~mol} \mathrm{~L}^{-1}$, time: $12 \mathrm{~h}$ ).

3.3.5 Reusability. The reusability is one of the important performances of IIPs, affecting their practical application. To test the reusability of the polymers, subsequent sorption and desorption cycles were performed using $0.0050 \mathrm{~g}$ IIP3 (NIP), in which $10 \mathrm{~mL} 20 \mathrm{mg} \mathrm{L}^{-1} \mathrm{Th}^{4+}$ in $1.0 \mathrm{~mol} \mathrm{~L}^{-1} \mathrm{HCl}$ was used in the adsorption process and $10 \mathrm{~mL} 0.1 \mathrm{~mol} \mathrm{~L}^{-1} \mathrm{Na}_{2}$ EDTA was applied in the leaching experiment. As shown in Fig. 10, the adsorption capacities of the IIP3 and NIP hardly decrease after five cycles, indicating that the $\mathrm{Th}^{4+}$-IIP3 (NIP) has good reusability in high acidity environment.

\section{Conclusions}

For the first time, a new $\mathrm{Th}^{4+}$-IIP that could be used in high acidity environment was synthesized using BMAOP as functional ligand. The molar ratio of the functional ligand to $\mathrm{Th}^{4+}$ was optimized to be 4 by ${ }^{31} \mathrm{P}$ NMR titration, elemental analysis, and EXAFS, which was verified by comparing the adsorption capacities and selectivities of $\mathrm{Th}^{4+}$-IIPs with different compositions. The best $\mathrm{Th}^{4+}$-IIP3, with the molar ratio of BMAOP to $\mathrm{Th}^{4+}$ at 4 , had a maximum adsorption capacity of $33.3 \mathrm{mg} \mathrm{g}^{-1}$ in $1 \mathrm{~mol} \mathrm{~L}^{-1}$ $\mathrm{HCl}$ solution, and its adsorption capacity was still considerable even in $6.0 \mathrm{~mol} \mathrm{~L}^{-1} \mathrm{HCl}$. The adsorption kinetic of the IIP3 followed the pseudo-second-order kinetic model. And the IIP3 can effectively extract $\mathrm{Th}^{4+}$ from the mixed aqueous solution of $\mathrm{Th}^{4+}$, $\mathrm{La}^{3+}, \mathrm{Eu}^{3+}, \mathrm{Yb}^{3+}, \mathrm{UO}_{2}{ }^{2+}$, and $\mathrm{Fe}^{3+}$. Moreover, the IIP3 can be reused for at least five times without obvious loss of adsorption capacity. Therefore, the prepared $\mathrm{Th}^{4+}$-IIP3 could be used in the detection and removal of $\mathrm{Th}^{4+}$ in high acidity waste water. Future studies will be focused on the improvement of the adsorption capacity and the selectivity coefficient $\left(\mathrm{Th}^{4+} / \mathrm{UO}_{2}{ }^{2+}\right)$ in high acidity environment for the treatment of the real samples.

\section{Acknowledgements}

The authors are grateful to Dr Zejun Li for the help in the ICPAES measurement. This work was supported by Science
Challenge Project (No. TZ2016004) and National Natural Science Foundation of China (No. U1507203 and 91226112).

\section{Notes and references}

1 S. C. Zhang, P. Liu and B. J. Zhang, World Nucl. Geosci., 2005, 22, 98-103.

2 Z. Gu, Chin. J. Nucl. Sci. Eng., 2007, 27, 97-105.

3 J. Cheng, Y. Hou and L. Che, Chin. Rare Earths, 2008, 29, 7677.

4 Z. Zhu, Y. Pranolo and C. Y. Cheng, Miner. Eng., 2015, 77, 185-196.

5 I. Shtangeeva, S. Ayrault and J. Jain, J. Environ. Radioact., 2005, 81, 283-293.

6 J. Fu, Q. D. Chen, T. X. Sun and X. H. Shen, Sep. Purif. Technol., 2013, 119, 66-71.

7 S. Kesava Raju Ch and M. S. Subramanian, J. Hazard. Mater., 2007, 145, 315-322.

8 T. P. Rao, P. Metilda and J. M. Gladis, Talanta, 2006, 68, 1047-1064.

9 R. Pilviö and M. Bickel, J. Alloys Compd., 1998, 271-273, 4953.

10 D. Nanda, M. S. Oak, M. P. Kumar, B. Maiti and P. K. Dutta, Sep. Sci. Technol., 2001, 36, 2489-2497.

11 W. A. Wan Ibrahim, L. I. Abd Ali, A. Sulaiman, M. M. Sanagi and H. Y. Aboul-Enein, Crit. Rev. Anal. Chem., 2014, 44, 233254.

12 C. Branger, W. Meouche and A. Margaillan, React. Funct. Polym., 2013, 73, 859-875.

13 J. Fu, L. Chen, J. Li and Z. Zhang, J. Mater. Chem. A, 2015, 3, 13598-13627.

14 H. L. Liang, Q. D. Chen and X. H. Shen, J. Nucl. Radiochem. Sci., 2016, 38, 129-144.

15 H. He, Q. Gan and C. Feng, RSC Adv., 2017, 7, 15102-15111.

16 Z. Zhang, J. Li, X. Song, J. Ma and L. Chen, RSC Adv., 2014, 4, 46444-46453.

17 F. F. He, H. Q. Wang, Y. Y. Wang, X. F Wang, H. S. Zhang, H. L. Li and J. H. Tang, J. Radioanal. Nucl. Chem., 2012, 295, 167-177.

18 A.-S. Chauvin, J.-C. G. Bünzli, F. Bochud, R. Scopelliti and P. Froidevaux, Chem.-Eur. J., 2006, 12, 6852-6864.

19 M. Monier and N. H. Elsayed, J. Colloid Interface Sci., 2014, 423, 113-122.

20 J. Fasihi, S. Ammari Alahyari, M. Shamsipur, H. Sharghi and A. Charkhi, React. Funct. Polym., 2011, 71, 803-808.

21 S. Buyuktiryaki, R. Say, A. Ersoz, E. Birlik and A. Denizli, Talanta, 2005, 67, 640-645.

22 C. Lin, H. Wang, Y. Wang and Z. Cheng, Talanta, 2010, 81, 30-36.

23 G. Huang, Z. Chen, L. Wang, T. Lv and J. Shi, J. Radioanal. Nucl. Chem., 2016, 310, 1265-1272.

24 J. Fasihi, N. Bavarsad, S. Shariati and K. Ashtari, Int. J. Environ. Anal. Chem., 2016, 96, 789-800.

25 E. Birlik, S. Buyuktiryaki, A. Ersoz, A. Denizli and R. Say, Sep. Sci. Technol., 2006, 41, 3109-3121.

26 Q. He, X. Chang, Q. Wu, X. Huang, Z. Hu and Y. Zhai, Anal. Chim. Acta, 2007, 605, 192-197. 
27 C. R. Lin, H. Q. Wang, Y. Y. Wang, L. Zhou and J. Liang, Int. J. Environ. Anal. Chem., 2011, 91, 1050-1061.

28 Z. Cheng, H. Wang, Y. Wang, F. He, H. Zhang and S. Yang, Microchim. Acta, 2011, 173, 423-431.

29 S. Mishra and D. K. Singh, Desalin. Water Treat., 2014, 56, 1364-1371.

30 G. X. Xu, Rare earths, Metallurgical Industry Press, Beijing, China, 2nd edn, 1995.

31 H. Tong, Y. Wang, W. Liao and D. Li, Sep. Purif. Technol., 2013, 118, 487-491.

32 S. Gao, T. X. Sun, Q. D. Chen and X. H. Shen, Radiochim. Acta, 2016, 104, 457-469.

33 S. Gao, X. H. Shen, Q. D. Chen and H. C. Gao, Sci. China: Chem., 2012, 55, 1712-1718.

34 K. Uezu, T. Kuwabara, M. Yoshida and G. Masahiro, Anal. Sci., 2004, 20, 1593-1597.

35 M. Yoshida, K. Uezu, M. Goto and S. Furusaki, Macromolecules, 1999, 32, 1237-1243.
36 F. H. El-Sweify, A. A. Abdel-Fattah and S. M. Ali, J. Chem. Thermodyn., 2008, 40, 798-805.

37 E. V. Goud, D. Das, A. Sivaramakrishna, K. Vijayakrishna, V. Sabareesh, G. Gopakumar, C. V. S. Brahmmananda Rao, M. Y. Lone and P. C. Jha, Polyhedron, 2016, 117, 741-748.

38 H. Yu, X. Wei, J. Li, S. Gu, S. Zhang, L. Wang, J. Ma, L. Li, Q. Gao, R. Si, F. Sun, Y. Wang, F. Song, H. Xu, X. Yu, Y. Zou, J. Wang, Z. Jiang and Y. Huang, Nucl. Sci. Tech., 2015, 26, 050102.

39 M. Newville, J. Synchrotron Radiat., 2001, 8, 322-324.

40 P. Metilda, J. Mary Gladis and T. Prasada Rao, Anal. Chim. Acta, 2004, 512, 63-73.

41 W. Meouche, K. Laatikainen, A. Margaillan, T. Silvonen, H. Siren, T. Sainio, I. Beurroies, R. Denoyel and C. Branger, Eur. Polym. J., 2017, 87, 124-135.

42 A. R. Iftikhar, H. N. Bhatti, M. A. Hanif and R. Nadeem, J. Hazard. Mater., 2009, 161, 941-947. 